\title{
VARIAÇÕES PLASMÁTICAS DE AMINAS VASOATIVAS EM EQUINOS SOB EFEITO DE SOBRECARGA DE CARBOIDRATOS E ANTI-INFLAMATÓRIOS NÃO ESTEROIDAIS
}

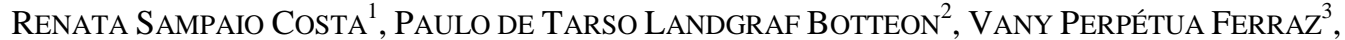

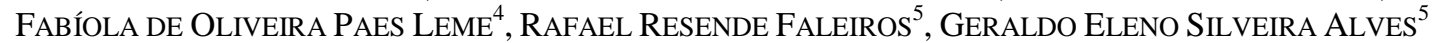 \\ ${ }^{1}$ Pós-graduanda em Medicina Veterinária na Murdoch University, Perth, Western Australia - renata_86@yahoo.com.br \\ ${ }^{2}$ Professor Doutor da Universidade Federal Rural do Rio de Janeiro, Seropédica, RJ, Brasil. \\ ${ }^{3}$ Químico da Universidade Federal de Minas Gerais, Belo Horizonte, MG, Brasil. \\ ${ }^{4}$ Professora Doutora da Pontifícia Universidade Católica do Rio Grande do Sul, Porto Alegre, RS, Brasil \\ ${ }^{5}$ Professor Doutor da Universidade Federal de Minas Gerais, Belo Horizonte, MG, Brasil
}

RESUMO

\begin{abstract}
Vinte equinos foram submetidos à sobrecarga de carboidratos (SC) e as variações plasmáticas das concentrações de serotonina (5-HT), putrescina (PUT) e cadaverina $(\mathrm{CAD})$ foram determinadas por cromatografia gasosa. Após 36h da SC, os animais foram divididos em quatro grupos $(n=5)$ e receberam, a cada $12 \mathrm{~h}$, por via intravenosa: solução salina $10 \mathrm{~mL}(\mathrm{GC})$, cetoprofeno 2,2mg/kg (GK), fenilbutazona 4,4 mg/kg (GF) e flunixina meglumina $1,1 \mathrm{mg} / \mathrm{kg}$ (GFM). Amostras de sangue foram coletadas em intervalos regulares após a SC $(0-72 \mathrm{~h})$. Às
\end{abstract}

6h após a SC observou-se um pico nas concentrações de 5-HT, com rápido retorno a valores abaixo dos basais. Não foram observadas variações nas concentrações de PUT; contudo, as concentrações de CAD se elevaram às 6h, assim permanecendo (com algumas variações) até o final do experimento. Conclui-se que a SC induz aumentos precoces (6h) nas concentrações plasmáticas de 5-HT e CAD. As concentrações plasmáticas das aminas estudadas não foram alteradas por nenhum dos tratamentos empregados.

PALAVRAS CHAVES: aminas vasoativas; anti-inflamatórios não esteroidais; equinos.

\section{PLASMATIC VARIATION OF VASOACTIVE AMINES IN HORSES UNDER EFFECT OF CARBOHYDRATE OVERLOAD AND NON-STEROIDAL ANTIINFLAMMATORIES}

Twenty horses were submitted to carbohydrate overload (CO) and plasmatic concentrations of the bioactive amine serotonin (5-HT), putrescin (PUT) and cadaverin (CAD) were determined by gas chromatography. After $36 \mathrm{~h}$ of carbohydrate overloading, horses were randomly distributed into four groups $(n=5)$ and were submitted to four intravenous treatments every 12 hours. The treatments were as follows: $10 \mathrm{ml}$ of saline (GC), ketoprofen $2.2 \mathrm{mg} / \mathrm{kg}$ (GK), phenylbutazone $4.4 \mathrm{mg} / \mathrm{kg}$ $(\mathrm{GF})$, and flunixin meglumine $1.1 \mathrm{mg} / \mathrm{kg}$ (GFM). Blood samples were collected at regular intervals $(0-72 \mathrm{~h})$ after the $\mathrm{CO}$. After $6 \mathrm{~h}$, there were peaks in the concentrations of 5-HT, which rapidly returned to values above the basal standard. No variations of PUT concentrations were observed; however CAD concentrations increased at $6 \mathrm{~h}$, remaining elevated (with some variation) until the end of the observation period. In conclusion, $\mathrm{CO}$ induced early (6h) increases in plasmatic concentrations of 5-HT and CAD. Plasmatic concentrations of the amines were not changed by any of the treatments used.

KEYWORDS: equine; NSAIDs; vasoactives amines. 


\section{INTRODUÇÃO}

Quando há fornecimento de quantidades elevadas de substrato fermentável, os microorganismos Gram-positivos no ceco proliferam-se e produzem ácido lático (GARNER et al., 1978). A descarboxilação dos aminoácidos resulta em pH baixo, logo, há aumento da produção de aminas (BEARSON et al., 1997). Algumas aminas produzidas pelas bactérias podem induzir vasoconstrição devido às suas semelhanças estruturais com aminas endógenas vasoconstritoras, como as catecolaminas (RANG \& DALE, 1991). A laminite aguda tem sido associada a alterações na digestão fermentativa no ceco, o que resulta em produção de substâncias vasoativas que afetam a circulação digital (GARNER et al., 1978).

As aminas bioativas (BA) são bases orgânicas alifáticas (putrescina, cadaverina, espermina, espermidina), alicíclicas (tiramina, feniletilamina) ou heterocíclicas (histamina, triptamina) (SILLA SANTOS, 1996).

Triptamina, tiramina e feniletilamina causam vasoconstrição em artérias e veias digitais in vitro, devido à estimulação de receptores de serotonina, liberação de noradrenalina ou pela liberação de serotonina das plaquetas (BAILEY et al., 2000; BAILEY et al., 2001). A triptamina é considerada a mais potente amina produzida no ceco, causando vasoconstrição, tanto in vitro quanto in vivo, por ativar, diretamente, os receptores de serotonina, com uma evidente seletividade pelas veias dos dígitos (ELLIOTT et al.,2003). Estudos prévios demonstraram aumento de triptamina, tiramina e feniletilamina em animais submetidos à sobrecarga de carboidratos para indução de laminite (BOTTEON et al. 2008).

Não se conhecem exatamente as funções da putrescina e cadaverina. Contudo, sabe-se que tais aminas estão presentes em concentrações elevadas nas células com alto metabolismo e renovação celular (LIMA \& GLORIA, 1999).

Este trabalho visou analisar as variações plasmáticas das aminas serotonina (5-HT), putrescina (PUT) e cadaverina (CAD) em equinos sob efeito de sobrecarga de carboidratos e o efeito terapêutico dos anti-inflamatórios não esteroidais, cetoprofeno, fenilbutazona e flunixina meglumina, iniciados 36h após a indução da sobrecarga.

\section{MATERIAL E MÉTODOS}

Foram utilizados 20 equinos hígidos (Equus caballus caballus), sem raça definida, machos castrados, com idade entre 3 e 15 anos, peso entre 264 e $344 \mathrm{~kg}$ e escore corporal de 2 a 3 (SPEIRS, 1997). Antes do experimento, os animais passaram por quarentena, período em que foram avaliados, pesados e numerados de 01 a 20 , sendo mantidos em piquete coletivo até sete dias antes de receberem a sobrecarga de carboidratos (SC) e com manutenção da oferta de água ad libitum, feno coast-cross (Cynodon dactylon), ração concentrada ${ }^{1}$ e $50 \mathrm{~g}$ de sal mineralizado para equinos, duas vezes ao dia, em quantidades equivalentes a $2,5 \%$ e $1 \%$ de peso corporal, respectivamente. Sete dias antes de receberem a SC, os animais foram privados da ração comercial e passaram por controle de endo e ectoparasitos, por meio de uma base conjugada de ivermectina e pamoato de praziquantel $^{2} \mathrm{e}$ deltametrina, respectivamente. Após a sobrecarga de carboidrato, os animais receberam suplemento eletrolítico $^{3}$ (25g/10L de água) adicionado diariamente à água de beber.

Os animais foram divididos, aleatoriamente, em quatro grupos experimentais $(n=5)$ e, 36h após receberem a SC, receberam, a cada $12 \mathrm{~h}$ por via intravenosa e de acordo com o grupo, $10 \mathrm{ml} \mathrm{de}$ solução salina $^{4}(\mathrm{GC}) ; 2,2 \mathrm{mg} / \mathrm{kg}$ de cetoprofeno ${ }^{5}$ (GK); $4,4 \mathrm{mg} / \mathrm{kg}$ fenilbutazona ${ }^{6}(\mathrm{GF}) ; \mathrm{e} 1,1 \mathrm{mg} / \mathrm{kg}$ de flunixina meglumina ${ }^{7}$ (GFM). A veia de administração utilizada foi a jugular esquerda, preservando-se a direita para a coleta das amostras de sangue em frascos a vácuo, contendo EDTA $^{8}$. As amostras foram colhidas antes da sobrecarga de carboidrato, 6 horas após a SC e a cada 12 horas até completar 72 horas de avaliação, ou seja, as amostras foram colhidas nos tempos $0 \mathrm{~h}, 6 \mathrm{~h}, 12 \mathrm{~h}, 24 \mathrm{~h}, 36 \mathrm{~h}$, 48h, 60h e $72 \mathrm{~h}$.

Após jejum alimentar e hídrico de $12 \mathrm{~h}$, os animais receberam amido de milho ${ }^{9}(20 \mathrm{~g} / \mathrm{kg})$, segundo modificação do modelo experimental referido por WEISS et al. (1998). Cada $\mathrm{kg}$ de amido foi dissolvido em um litro de água, sendo administrado via sonda nasogástrica $(11 \mathrm{~mm})$. Imediatamente após a administração do amido, os animais voltaram a receber o feno.

Para a extração das aminas vasoativas, foram utilizadas amostras com 1,5 mL de plasma e foi promovida a precipitação de proteínas por meio da adição de $100 \mu \mathrm{l}$ de solução de ácido

\footnotetext{
${ }^{1}$ Nutriage-Guabi (15\% de proteína). Brasil

${ }^{2}$ Centurion-Valleé. Brasil.

${ }^{3}$ Plusvital.Marcolab. Brasil.

4 Solução salina 0,9\%-Sanobiol. Brasil.

${ }^{5}$ Ketofen- Merial. Brasil.

${ }^{6}$ Equipalazone- Marcolab. Brasil.

${ }^{7}$ Flumegan- Marcolab. Brasil

${ }^{8}$ Vacutainer. Bacton \& Dickinson. Brasil.

${ }^{9}$ Milhena- Nutril. Brasil
} 
trifluoroacético $^{10}$ a $10 \mathrm{moL}$. As amostras foram então centrifugadas ${ }^{11}$ a $12.000 \mathrm{rpm}$, por 15 minutos. Uma alíquota de $0,2 \mathrm{~mL}$ do sobrenadante foi transferida para tubos criogênicos ${ }^{12}$, submetida à liofilização e armazenada em freezer a $-20^{\circ} \mathrm{C}$. O material liofilizado foi ressuspendido em $100 \mu \mathrm{L}$ de solução de anidrido trifluoroacético/acetonitrila na proporção de 1:1. Essa solução foi homogeneizada em vórtex e aquecida no microondas ${ }^{13}$ por 3 min. Os derivados acilados foram secos em linha de nitrogênio e, em seguida, retomados com $50 \mu \mathrm{L}$ de acetonitrila marcada com padrão interno ${ }^{14} ; 2 \mu \mathrm{L}$ dessa solução foram colocados no cromatógrafo ${ }^{15}$ para análise.

As condições de operação do cromatógrafo gasoso foram mantidas a $150^{\circ} \mathrm{C}, 1 \mathrm{~min}, 7^{\circ} \mathrm{C} / \mathrm{min}$, $220^{\circ} \mathrm{C}, 20^{\circ} \mathrm{C} / \mathrm{min}, 270^{\circ} \mathrm{C}$, injetor e detector a $280^{\circ} \mathrm{C}$, Split 1/100, sendo utilizada a coluna capilar ${ }^{16}$. Utilizou-se o hidrogênio como gás de arraste.

As concentrações obtidas de serotonina apresentaram dispersão não normal e não foi possível a transformação das variáveis; portanto, os dados foram analisados pelo teste de Kruskal-Wallis com nível de significância de 5\%. As médias foram submetidas a comparações múltiplas pareadas utilizando-se o procedimento de Dunn/ Teste bilateral. Já as concentrações de putrescina e cadaverina apresentaram dispersão normal e os dados foram analisados pelo teste de análise de variância e as médias foram submetidas ao teste de Tukey.

\section{RESULTADOS E DISCUSSÃO}

A produção de aminas por bactérias é influenciada pelo $\mathrm{pH}$ do meio, concentração de aminoácidos livres e de carboidratos fermentáveis (LIMA \& GLORIA, 1999). Em meios ácidos, a produção de aminas é estimulada, visto que nessas condições de $\mathrm{pH}$ as descarboxilases têm melhor nível de atividade. Devido às suas similaridades estruturais com aminas endógenas, muitas das aminas encontradas no conteúdo cecal de equinos, se liberadas, podem exercer efeitos na vasculatura (RANG \& DALE, 1991). Apesar de a fisiopatologia da laminite ainda não estar completamente esclarecida, a isquemia digital é considerada um dos eventos que acarreta a ocorrência de tal afecção (HOOD et al., 2001).

\footnotetext{
${ }^{10}$ Acido Tricloroacético PA/ACS -Cristal (marca Synth)

${ }^{11}$ 5415D Eppendorf

${ }^{12}$ Tubo criogênico c/ tampa de rosca, estéril (marca Alfa).

${ }^{13}$ Panasonic Píccolo $600 \mathrm{~W}$.

${ }^{14} \mathrm{C} 18$ - Octadecano $0,74 \mathrm{~g} / \mathrm{L}$

${ }^{15}$ Cromatógrafo a gás Varian CP-3380

${ }^{16}$ BP10 ( $14 \%$ cyanopropylphenyl dimethyl siloxane) $25 \mathrm{~m} \mathrm{x} 0.22 \mathrm{~mm}$ 0,25 um (SGE)
}

A concentração plasmática de serotonina (5HT) apresentou um pico no tempo $6 \mathrm{~h}$ em todos os grupos analisados (Tabela 1). Deve-se observar, principalmente, a 5-HT no GK, cuja concentração no momento $6 \mathrm{~h}$ foi estatisticamente superior ao momento 0 .

O aumento da concentração de serotonina pode ser explicado devido à sobrecarga de carboidratos. Segundo FERNSTROM \& WURTMAN (1971), a ingestão de carboidratos acarreta aumento indireto nas concentrações do aminoácido precursor da serotonina, o triptofano. Embora não haja aminoácidos nos carboidratos, quando eles são ingeridos, há liberação de insulina que causa aumento da concentração de triptofano, logo, há aumento da produção de serotonina.

A serotonina, ou 5-Hidroxitriptamina (5HT), é uma amina biogênica conhecida, principalmente, por seu papel como neurotransmissor. Ela é uma molécula com diversos efeitos no sistema nervoso central e no periférico, agindo como hormônio, neurotransmissor etc. A 5HT possui papel relevante na modulação da motilidade gastrointestinal e está envolvida na manutenção do tônus vascular periférico, tônus vascular cerebral, no funcionamento das plaquetas, tendo sido relacionada com a fisiopatologia de desordens de humor, de comportamento, de apetite, hipertensão etc. (KANDEL, 2001).

Segundo KANDEL (2001), depressão, manias, ansiedade, alterações comportamentais, entre outros tipos de desordens, estão associadas à diminuição da concentração de 5-HT disponível no SNC. Observou-se, no presente estudo, o aumento da concentração de 5-HT.

Entretanto, pode-se pensar nos efeitos deletérios causados pelo aumento excessivo de serotonina no organismo. Sabe-se que a 5-HT possui papel importante na amplificação da agregação plaquetária. Mesmo não existindo lesão no endotélio, as plaquetas podem se aderir e promover agregação sobre o endotélio íntegro (ROSENBLUM, 1997; REININGER, et al., 1998), levando à formação de trombos, por exemplo. Em estudo realizado por PAES LEME et al. (2006), foi observado que a ativação plaquetária inicia-se $6 \mathrm{~h}$ após a administração do carboidrato e que os antiinflamatórios não-esteroidais reduzem a ativação das plaquetas de equinos in vivo, sendo que a flunixina meglumina apresenta os melhores resultados.

Os efeitos vasoconstrictores da 5-HT também são relevantes. Segundo ELLIOTT et al. (2003), a serotonina pode ser liberada pelas plaquetas ativadas, induzindo vasoconstrição em grandes artérias, veias e vênulas. Além disso, ela amplifica a ação de outras substâncias, como 
noradrenalina, histamina etc. Aminas como a triptamina, tiramina e feniletilamina, também analisadas no presente estudo, causam vasoconstrição em artérias e veias digitais in vitro, pela estimulação de receptores de serotonina ou pela liberação de serotonina das plaquetas (BAILEY et al., 2000; BAILEY et al., 2001).

Tabela 1 - Médias das concentrações plasmáticas de serotonina, em diferentes tempos (h), de equinos sob efeitos de sobrecarga de carboidratos e de anti-inflamatórios não esteroidais

\begin{tabular}{cllcccccc}
\hline Tempo(h) & \multicolumn{2}{c}{ GK } & \multicolumn{2}{c}{ GF } & \multicolumn{2}{c}{ GFM } & \multicolumn{2}{c}{ GC } \\
\hline 0 & 8,2 & $\mathrm{Ba}$ & 35,7 & $\mathrm{Aa}$ & 19,4 & $\mathrm{Aa}$ & 38,8 & $\mathrm{Aa}$ \\
6 & 105,7 & $\mathrm{Aa}$ & 100,5 & $\mathrm{Aa}$ & 67,9 & $\mathrm{Aa}$ & 45,3 & $\mathrm{Aa}$ \\
12 & 1,9 & $\mathrm{Ca}$ & 25,9 & $\mathrm{Ba}$ & 29,7 & $\mathrm{Ba}$ & 77,1 & $\mathrm{Aa}$ \\
24 & 0,4 & $\mathrm{Da}$ & 0,0 & $\mathrm{Da}$ & 0,1 & $\mathrm{Da}$ & 0,3 & $\mathrm{Ca}$ \\
36 & 1,9 & $\mathrm{Ca}$ & 0,5 & $\mathrm{Da}$ & 0,2 & $\mathrm{Da}$ & 0,1 & $\mathrm{Ca}$ \\
48 & 0,0 & $\mathrm{Da}$ & 0,7 & $\mathrm{Da}$ & 0,4 & $\mathrm{Da}$ & 0,1 & $\mathrm{Ca}$ \\
60 & 2,6 & $\mathrm{Ca}$ & 5,3 & $\mathrm{Ca}$ & 1,7 & $\mathrm{Ca}$ & 2,8 & $\mathrm{Ba}$ \\
72 & 4,4 & $\mathrm{Ba}$ & 5,8 & $\mathrm{Ca}$ & 4,2 & $\mathrm{Ca}$ & 4,9 & $\mathrm{Ba}$ \\
\hline
\end{tabular}

Médias seguidas de diferentes letras maiúsculas (nas colunas) e minúsculas (nas linhas) diferem significativamente (P=0,0001) pelo teste de Kruskal-Wallis. Observam-se as variações plasmáticas de 5-HT nos tempos avaliados e em relação aos anti-inflamatórios cetoprofeno (Ketofen- Merial. Brasil) (GK) 2,2mg/kg, fenilbutazona (Equipalazone- Marcolab. Brasil) (GF) 4,4mg/kg, flunixina meglumina (Flumegan- Marcolab. Brasil) (GFM) 1,1mg/kg e solução salina (Solução salina 0,9\%- Sanobiol. Brasil) (GC) 10mL.

Segundo BAILEY et al. (2002), em estudo realizado in vitro, a concentração de putrescina (PUT) no conteúdo cecal de equinos encontra-se aumentada quando há sobrecarga de carboidrato. Cerca de 1L de conteúdo cecal de equinos foi mantido sob condições de anaerobiose, adicionaramse diferentes concentrações de carboidrato ao meio e o conteúdo cecal foi analisado utilizando-se a cromatografia líquida de alta eficiência (HPLC). Aminas, entre elas, a putrescina, foram identificadas e suas concentrações mensuradas. Observou-se aumento da concentração de putrescina após $18 \mathrm{~h}$ de incubação. No presente estudo, tal fato não foi constatado, pois não foram observadas variações nas concentrações de PUT nos diferentes grupos e tempos avaliados (Tabela 2). Tal diferença nos resultados pode ser devida às diferenças metodológicas, pois o presente trabalho foi realizado in vivo e o método de análise foi a cromatografia gasosa.

Observou-se que no tempo $72 \mathrm{~h}$ houve diminuição significativa da concentração de PUT apenas no GC. Esse resultado pode indicar a possível ação, ainda não esclarecida, dos anti-inflamatórios sobre as concentrações plasmáticas dessa amina.

Tabela 2 - Médias das concentrações plasmáticas de putrescina, em diferentes tempos (h), de equinos sob efeitos de sobrecarga de carboidratos e de anti-inflamatórios não esteroidais

\begin{tabular}{ccccc}
\hline Tempo(h) & GK & GF & GFM & GC \\
\hline 0 & $8,7 \mathrm{Aa}$ & $4,1 \mathrm{Aa}$ & $3,3 \mathrm{Aa}$ & $13,1 \mathrm{Aa}$ \\
6 & $3,1 \mathrm{Aa}$ & $1,9 \mathrm{Aa}$ & $2,1 \mathrm{Aa}$ & $1,5 \mathrm{Ba}$ \\
12 & $3,2 \mathrm{Aa}$ & $3,2 \mathrm{Aa}$ & $3,6 \mathrm{Aa}$ & $5,1 \mathrm{ABa}$ \\
24 & $1,6 \mathrm{Aa}$ & $1,9 \mathrm{Aa}$ & $1,3 \mathrm{Aa}$ & $1,4 \mathrm{Ba}$ \\
36 & $2,4 \mathrm{Aa}$ & $0,5 \mathrm{Aa}$ & $5,4 \mathrm{Aa}$ & $1,9 \mathrm{Ba}$ \\
48 & $2,4 \mathrm{Aa}$ & $1,4 \mathrm{Aa}$ & $1,5 \mathrm{Aa}$ & $0,9 \mathrm{Ba}$ \\
60 & $3,2 \mathrm{Aa}$ & $4,8 \mathrm{Aa}$ & $2,3 \mathrm{Aa}$ & $4,6 \mathrm{ABa}$ \\
72 & $1,6 \mathrm{Aa}$ & $0,6 \mathrm{Aa}$ & $0,5 \mathrm{Aa}$ & $0,5 \mathrm{Ba}$ \\
\hline
\end{tabular}

Médias seguidas de diferentes letras maiúsculas (nas colunas) diferem significativamente pelo teste de análise de variância $(\mathrm{P}=$ $0,001)$ e médias seguidas de diferentes letras minúsculas (nas linhas) não diferem significativamente pela $\mathrm{ANOVA}(\mathrm{P}=0,48)$. Observam-se variações plasmáticas da PUT nos tempos avaliados e em relação aos anti-inflamatórios cetoprofeno(Ketofen- Merial. Brasil) (GK) 2,2mg/kg, fenilbutazona(Equipalazone- Marcolab. Brasil) (GF) 4,4mg/kg, flunixina meglumina(Flumegan- Marcolab. Brasil) (GFM) 1,1mg/Kg e solução salina (Solução salina 0,9\%- Sanobiol. Brasil) (GC) $10 \mathrm{~mL}$. 
Quanto à cadaverina (CAD), observou-se que as concentrações apresentaram-se instáveis durante os tempos avaliados (Tabela 3 ).

Um fato interessante foi observado ao se analisar o GC. Verificou-se que a concentração de $\mathrm{CAD}$ no tempo $72 \mathrm{~h}$ retornou aos valores basais (tempo 0h) apenas no GC. Tal fato pode ser indicativo de que há efeitos dos anti-inflamatórios não esteroidais sobre a concentração plasmática de cadaverina. Para o esclarecimento desse achado, há necessidade de que mais pesquisas sejam realizadas.

$\mathrm{Na}$ literatura consultada, putrescina e cadaverina foram as aminas de menor citação e ainda não se conhece, ao certo, suas funções; contudo, sabe-se que estão envolvidas no processo de proliferação e manutenção da viabilidade celular (COHEN, 1998; LIMA \& GLORIA, 1999).

Tabela 3 - Médias das concentrações plasmáticas de cadaverina, em diferentes tempos (h), de equinos sob efeitos de sobrecarga de carboidratos e de anti-inflamatórios não esteroidais

\begin{tabular}{crrrr}
\hline Tempo(h) & GK & GF & GFM & GC \\
\hline 0 & $1,3 \mathrm{Ba}$ & $3,2 \mathrm{Ba}$ & $9,3 \mathrm{Ca}$ & $1,9 \mathrm{Ba}$ \\
6 & $17,2 \mathrm{Aa}$ & $20,2 \mathrm{Aa}$ & $17,5 \mathrm{Ba}$ & $19,8 \mathrm{Aa}$ \\
12 & $11,9 \mathrm{Aa}$ & $6,7 \mathrm{Ba}$ & $65,9 \mathrm{Aa}$ & $10,9 \mathrm{Aa}$ \\
24 & $3,3 \mathrm{Ba}$ & $24,2 \mathrm{Aa}$ & $21,7 \mathrm{Ba}$ & $21,8 \mathrm{Aa}$ \\
36 & $18,1 \mathrm{Aa}$ & $2,4 \mathrm{Ba}$ & $3,9 \mathrm{Ca}$ & $4,7 \mathrm{Ba}$ \\
48 & $20,1 \mathrm{Aa}$ & $18,7 \mathrm{Aa}$ & $19,3 \mathrm{Ba}$ & $17,7 \mathrm{Aa}$ \\
60 & $0,9 \mathrm{Ba}$ & $16,9 \mathrm{Aa}$ & $2,1 \mathrm{Ca}$ & $11,5 \mathrm{Aa}$ \\
72 & $14,4 \mathrm{Aa}$ & $14,7 \mathrm{Aa}$ & $42,2 \mathrm{Aa}$ & $5,0 \mathrm{Ba}$ \\
\hline
\end{tabular}

\section{CONCLUSÃO}

Conclui-se que a sobrecarga de carboidrato induz aumentos precoces $(6 \mathrm{~h})$ nas concentrações plasmáticas de serotonina (5-HT) e cadaverina (CAD).

As concentrações plasmáticas das aminas estudadas (serotonina, putrescina e cadaverina) não foram alteradas por nenhum dos tratamentos (cetoprofeno, fenilbutazona e flunixina meglumina).

\section{AGRADECIMENTOS}

À Fundação de Amparo à Pesquisa do Estado de Minas Gerais (FAPEMIG) e ao Conselho Nacional de Desenvolvimento Científico e Tecnológico $(\mathrm{CNPq})$ pela bolsa de Iniciação Científica e o suporte financeiro.

\section{COMITÊ DE ÉTICA E BIOSSEGURANÇA} 137/2008.

Protocolo de aprovação CETEA/UFMG: $n^{\circ}$

\section{REFERÊNCIAS}

BAILEY, S.R., CUNNINGHAM, F.M., ELLIOTT, J. Endotoxin and dietary amines may increase plasma 5- hydroxytryptamine in the horse. Equine Veterinary Journal, v.32, n.6, p.497-504, 2000.

BAILEY, S. R, BERHANE, Y., MARR, C. M, ELLIOTT, $\mathrm{J}$. Amines from the equine hindgut may cause digital vasoconstriction by direct or indirect mechanisms. Journal of Veterinary Internal Medicine, v.15, n.3, p.379, 2001.

BAILEY, S.R., RYCROFT, A., ELLIOTT, J. Production of amines in equine cecal contents in an in vitro model of carbohydrate overload. Journal of Animal Science, v.80, p.2656-2662, 2002.

BAILEY, S.R., KATZ, L.M., BERHANE, Y., SAMUELS, T., DE BRAUVERE, N., MARR, C.M., ELLIOT, J. Seasonal changes in plasma concentrations of cecum-derived amines in clinically normal ponies predisposed to laminits. American Journal of Veterinary Research, v.64, p.1132-1138, $2003 \mathrm{~b}$.

BOTTEON, P.T.L., FERRAZ, V., GLORIA, M.B.A., MELO, M.M, PAES LEME, F.O, FALEIROS, R.R., ALVES, G.E.S. Concentrações plasmáticas de triptamina, tiramina e feniletilamina em eqüinos sob efeitos de sobrecarga de carboidratos e antiinflamatórios não esteroidais. Pequisa Veterinária Brasileira, v.28, p.299302, 2008.

BEARSON, S., BEARSON, B. Acid stress responses in enterobacteria. FEMS Microbiology Letters, v.47, n.2, p.173-180, 1997.

COHEN, S.S. A guide to the polyamines. Oxford University Press, Current Pharmaceutical Design, v.12, n.23, p.2923-2934, 1998. 
ELLIOTT, J., BERHANE, Y., BAILEY, S.R. Effects of monoamines formed in the cecum of horses on equine digital blood vessels and platelets. American Journal of Veterinary Research, v.64, n.9, p.1124-1131, 2003.

FERNSTROM, J.D E WURTMAN, R.J. Brain serotonin content: increase following ingestion of a carbohydrate diet. Science, v. 174, p. 1023-1025, 1971.

GARNER, H. E., MOORE, J. N., JOHNSON, J.H., CLARK, L., AMEND, J. F., TRITSCHLER, L.G., COFFMAN, J.F., SPROUSE, R.F., HUTCHESON, D.P. AND SALEM, C.A. Changes in the caecal flora associated with the onset of laminitis Equine Veterinary Journal, v.10, p.249-252, 1978.

HOOD, D.M., WAGNER, I.P., BRUMBAUGH, G.W. Evaluation of hoof wall surface temperature as an index of digital vascular perfusion during the prodromal and acute phases of carbohydrate-induced laminitis in horses. American J. of Veterinary Research, v.62, n.7, p.11671172, 2001.

KANDEL, E. Depression, mania and anxiety disorders. In Principles of Neural Science. McGraw-Hill, New York, 4th edn., p. 1209-1225, 2001.

LIMA, A.S., GLORIA, M.B.A. Aminas bioativas em alimentos. Boletim da Sociedade Brasileira de Ciência e Tecnologia de Alimentos, v.33, n.1, p.70-79, 1999.

PAES LEME, F.O., WURZINGER, L.J.; VASCONCELOS, A.C. AND ALVES, G.E.S. Ativação de plaquetas de eqüinos com laminite induzida e tratados com ketoprofeno, fenilbutazona e flunixin meglumina.
Arquivo Brasileiro de Medicina Veterinária e Zootecnia, vol.58, n.2, pp. 149-157, 2006.

RANG, H.P., DALE, M.M. Adrenergic transmission. In: Pharmacology, 2 ed. Churchill Livingston, London, UK, p.212, 1991.

REININGER, A., KORNDÖRFER, M.A, WURZINGER, L.J. Adhesion of ADP- activated platelets to intact endothelium under stagnation point flow in vitro is mediated by Integrin alIbb3. Journal of Thrombosis and Haemostasis, v.70, p.998-1003, 1998.

ROSENBLUM, W.I. Platelet adhesion and aggregation without endothelial denudation or exposure of basal lamina and/or collagen. Journal of Vascular Research, v.34, p.409-417, 1997.

SILLA SANTOS, M. H. Biogenic amines: their importance in foods. International Journal Food Microbiology, v. 29, p. 213-231. 1996.

SPEIRS, V.C. The alimentary tract. Clinical examination of horses. Philadelphia: W.B. Saunders, p.261-298, 1997.

WEISS, D.J., EVANSON, O.A., GREEN, B.T., BROWN, D.R. In vitro: evaluation of intraluminal factors that may alter intestinal permeability in ponies with carbohydrateinduced laminitis. American Journal of Veterinary Research, v.61, p.858- 861, 2000.

WEISS, D.J., EVANSON, O.A., MCCLENAHAN, D., FAGLIARI, J.J,DUNNWIDDIE, C.T., WELLS, R.E. Effect of a competitive inhibitor of platelet aggregation on experimentally induced laminitis in ponies. American Journal of Veterinary Research, v.59, p.814-817, 1998. 